EPJ Web of Conferences 71, 00023 (2014)

DOI: 10.1051/epjconf/20147100023

(C) Owned by the authors, published by EDP Sciences, 2014

\title{
Top Quark Pair Properties - Spin Correlation, Charge Asymmetry, and Complex Final States - at ATLAS
}

\author{
Elizabeth Brost ${ }^{1, a}$, On behalf of the ATLAS Collaboration \\ ${ }^{1}$ University of Oregon, 1585 E. 13th Avenue, Eugene Oregon, 97403 USA
}

\begin{abstract}
We present measurements of top quark pair properties performed with the ATLAS detector at the Large Hadron Collider in proton-proton collisions at a centerof-mass energy of $\sqrt{s}=7 \mathrm{TeV}$. The latest measurements of spin correlation and charge asymmetry in $t \bar{t}$ events, as well as measurements of the cross section for $t \bar{t}$ production in association with vector bosons, are presented.
\end{abstract}

\section{Introduction}

The top quark is the heaviest known fundamental particle. Top quarks decay before hadronization, in $O\left(0.5 \times 10^{-24} \mathrm{~s}\right)[1]$. Thus, in studying the top quark, we are given a rare opportunity to study a bare quark. Measurements of top quark properties can help in testing the Standard Model (SM), as well as give us a handle on new physics.

Top quark pair production at the Large Hadron Collider (LHC) occurs predominantly through gluon-gluon fusion $(g g \rightarrow t \bar{t})$, approximately $80 \%$ of the time [1]. Top quarks decay to a $W$ boson and a bottom quark nearly $100 \%$ of the time in the Standard Model. Top-antitop events are classified into three decay signatures for analysis: dilepton events (where both $W$ bosons decay leptonically), fully hadronic events (where both $W$ bosons decay hadronically), and single lepton events (where one $W$ boson decays leptonically and the other decays hadronically).

The measurements described here were performed using some or all of the $4.7 \mathrm{fb}^{-1}$ of $\sqrt{s}=7$ $\mathrm{TeV}$ data collected in 2011 by the ATLAS experiment [2] at the LHC.

\section{Spin Correlation}

The spins of the top and antitop quarks in $t \bar{t}$ events are expected to be correlated in the Standard Model (SM) [3]. Since the top quarks will decay before their spins have time to flip, the spin information will be passed on to the decay products. In events where the top pair is produced through the fusion of like-helicity gluons, we therefore expect that the charged leptons will have a spin correlation in the azimuthal angle, $\phi$. We can then use the opening angle $(\Delta \phi)$ distribution of the two leptons to compute the degree of correlation between the spins of the top and antitop quarks.

Using $2.1 \mathrm{fb}^{-1}$ of $\sqrt{s}=7 \mathrm{TeV}$ proton-proton data collected in 2011, we are able to observe spin correlations in top-antitop events for the first time [4]. Candidate events are selected with two leptons (electrons or muons), missing transverse energy, and at least two jets.

a e-mail: ebrost@uoregon.edu

This is an Open Access article distributed under the terms of the Creative Commons Attribution License 2.0, which permits unrestricted use, distribution, and reproduction in any medium, provided the original work is properly cited. 
The degree of correlation,

$$
A=\frac{N(\uparrow \uparrow)+N(\downarrow \downarrow)-N(\uparrow \downarrow)-N(\downarrow \uparrow)}{N(\uparrow \uparrow)+N(\downarrow \downarrow)+N(\uparrow \downarrow)+N(\downarrow \uparrow)},
$$

is defined as the fractional difference between the number of events where the top and antitop spins are aligned and those where the spins are anti-aligned. The measured degree of correlation depends not only on the $t \bar{t}$ production mechanism, but also on the choice of the basis in which the spins are measured. The two bases considered in this analysis are the helicity basis, $A_{\text {helicity }}$, which is chosen to be the direction of flight of the top quark in the rest frame of the $t \bar{t}$ system, and the maximal basis, $A_{\text {maximal }}$, which is optimized for top pair production by $g g$ fusion, as described in [5].

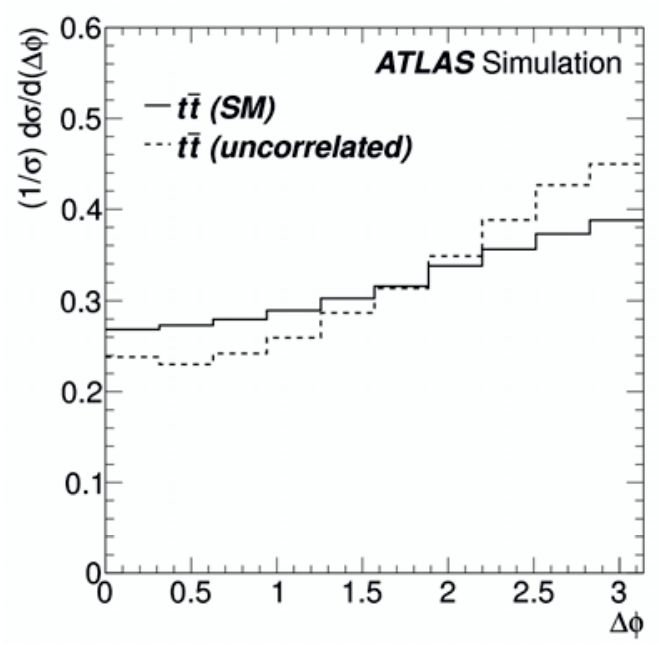

Figure 1. Normalized $\Delta \phi$ distribution for charged leptons in Standard Model (solid line) and uncorrelated (dashed line) scenarios [4].

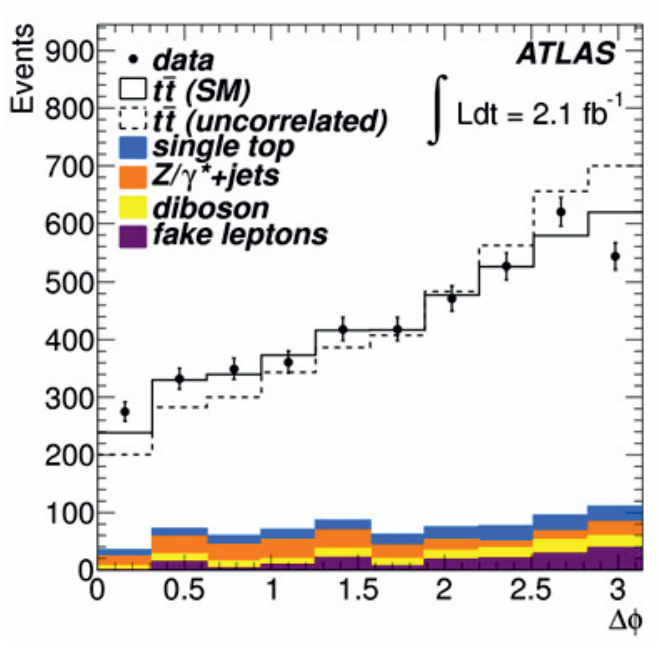

Figure 2. Measured $\Delta \phi$ distribution in all three channels $(e e, e \mu$, and $\mu \mu$ ), along with normalized $\mathrm{MC}$ background samples. Also shown are Standard Model (solid line) and uncorrelated (dashed line) $t \bar{t}$ samples [4].

Figure 1 shows the $\Delta \phi$ distribution for the pair of charged leptons, for two $t \bar{t}$ Monte Carlo samples. In the first (solid line), the spins of the top and antitop quarks are correlated as in the Standard Model, while in the second (dashed line), the spins are completely uncorrelated. These samples were generated using MC@NLO [6] and the CTEQ6.6 parton distribution function (PDF) set, and hadronized with HERWIG [7].

Figure 2 shows the measured lepton $\Delta \phi$ distribution in data, overlaid on the two $t \bar{t}$ samples and relevant backgrounds. The spin correlation in data is extracted from the lepton $\Delta \phi$ distribution using a binned log-likelihood fit. The fit includes a superposition of the correlated and uncorrelated simulations, with a multiplicative factor, $f^{S M} . f^{S M}$ is the degree to which the data fit the SM template. A value of $f^{S M}=0$ indicates no correlation between the spins of the top and antitop quarks, while $f^{S M}$ $>1$ indicates that the spins are more correlated than predicted by the SM.

In this dataset, $f^{S M}$ is measured to be $1.30 \pm 0.14$ (stat.) ${ }_{-0.22}^{+0.27}$ (syst.). We can thus exclude the zero-correlation hypothesis at $5.1 \sigma$. 
We can interpret the measured value of $f^{S M}$ in terms of $A_{\text {basis }}$ in a chosen basis. The value $A_{\text {basis }}^{\text {measured }}$ is given through the relation $A_{\text {basis }}^{\text {measured }}=A_{\text {basis }}^{S M} \cdot f^{S M}$. The SM predictions for spin correlation in $t \bar{t}$ events are $A_{\text {helicity }}^{S M}=0.31$ and $A_{\text {maximal }}^{S M}=0.44$, for the helicity and maximal bases, respectively. In the helicity basis, the measured degree of correlation is $A_{\text {helicity }}^{\text {mearured }}=0.40 \pm 0.04$ (stat.) ${ }_{-0.07}^{+0.08}$ (syst.), and in the maximal basis, $A_{\text {maximal }}^{\text {measured }}=0.57 \pm 0.06$ (stat.) ${ }_{-0.10}^{+0.12}$ (syst.). The largest uncertainties in this analysis originate from uncertainties on fake leptons, and the jet energy scale, resolution, and efficiency. This is the first measurement of $t \bar{t}$ spin correlation made at the LHC.

\section{Charge Asymmetry}

Top pair production at hadron colliders is expected to be symmetric at leading order, under exchange of the top and antitop quarks. While top pair production via gluon-gluon fusion is symmetric, the contributions from the $q \bar{q} \rightarrow t \bar{t}$ and $q g \rightarrow t \bar{t}$ processes are antisymmetric. Thus, $t \bar{t}$ charge asymmetry should be non-zero (but small) at the LHC. In addition to these SM processes, however, beyondthe-Standard Model (BSM) processes could alter that asymmetry through the addition of anomalous vector or axial-vector couplings, or through interference with the SM, giving us insight into new physics.

Thus, we measure the top pair charge asymmetry in $t \bar{t}$ events,

$$
A_{C}=\frac{N(\Delta|y|>0)-N(\Delta|y|<0)}{N(\Delta|y|>0)+N(\Delta|y|<0)},
$$

where $\Delta|y| \equiv\left|y_{t}\right|-\left|y_{\bar{t}}\right|$ is the difference between the absolute values of the rapidities of the top and antitop quarks and $N$ is the number of events where that quantity is positive or negative. The Standard Model predicts a small $t \bar{t}$ charge asymmetry of $A_{C}^{S M}=0.0123 \pm 0.0005$ [8] (computed at next-toleading order with electroweak corrections) at the LHC.

\subsection{Single Lepton + Jets Channel}

Using $4.7 \mathrm{fb}^{-1}$ of $\sqrt{s}=7 \mathrm{TeV}$ data collected in 2011, semileptonic $t \bar{t}$ candidate events are selected with one high- $p_{T}$, isolated lepton (electron or muon), missing transverse energy, and at least four jets (at least one of which is $b$-tagged). Jets originating from $b$-quark hadronization are identified using a multivariate algorithm that makes use of impact parameter significance, primary vertex information, and the topology of decay products inside the jet.

A kinematic fit is used to reconstruct the candidate events, and then the reconstructed $\Delta|y|$ distribution (shown in Figure 3) is unfolded to the parton level using the Fully Bayesian Unfolding technique [10], to correct for detector effects.

The charge asymmetry is measured to be $A_{C}=0.006 \pm 0.010$ (stat. + syst.). In addition to the inclusive measurement of charge asymmetry, differential measurements as a function of $t \bar{t}$ invariant mass are also presented, as shown in Figure 4, along with predictions from the SM and two color-octet massive axigluon models.

No deviations from the Standard Model are seen, and all measurements made are consistent with the SM predictions, within the uncertainties. 


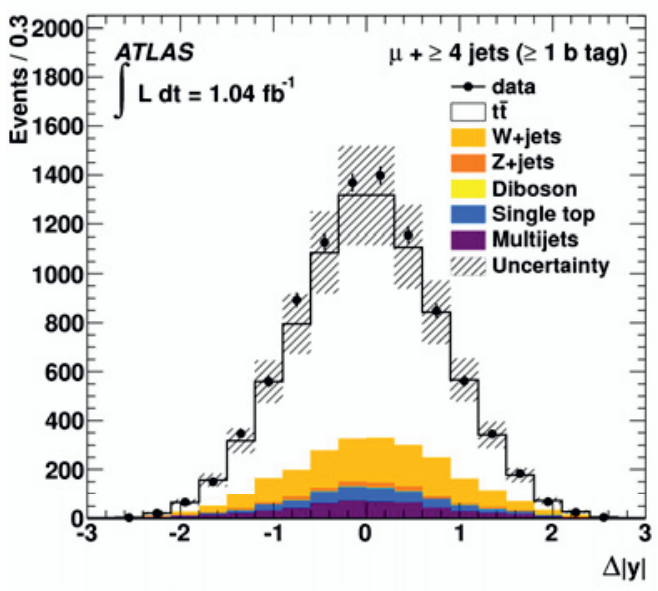

Figure 3. Measured $\Delta|y|$ distribution for the combined electron and muon channels. Data (black points) and Monte Carlo predictions (solid colors) are shown [9].

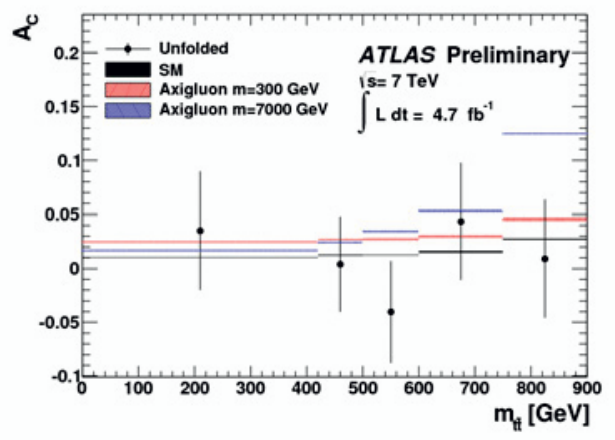

Figure 4. $A_{C}$ values as a function of $m_{t \bar{t}}$ are shown (black dots), along with the Standard Model predictions (black), and predictions from models with coloroctet axigluons with mass $\mathrm{m}=300 \mathrm{GeV}$ (red) or $\mathrm{m}=$ $7000 \mathrm{GeV}$ (blue) [9].

\subsection{Dilepton Channel}

Using $4.7 \mathrm{fb}^{-1}$ of $\sqrt{s}=7 \mathrm{TeV}$ data collected in 2011, candidate dilepton $t \bar{t}$ events are selected with two oppositely-charged leptons (electrons or muons), missing transverse energy, and at least two jets. The event is then reconstructed by computing the leading-order matrix element for the $g g \rightarrow t \bar{t}$ process. Having reconstructed the top and antitop quarks, the $t \bar{t}$ charge asymmetry $\left(A_{C}^{t \bar{t}}\right)$ is evaluated as in Section 3.

We also present a lepton-based asymmetry for these events,

$$
A_{C}^{\ell \ell}=\frac{N(\Delta|\eta|>0)-N(\Delta|\eta|<0)}{N(\Delta|\eta|>0)+N(\Delta|\eta|<0)}
$$

where $\Delta|\eta| \equiv\left|\eta_{\ell^{+}}\right|-\left|\eta_{\ell^{-}}\right|$is the difference between the absolute values of the pseudorapidities of the postively-charged and the negatively-charged leptons, and $N$ is the number of events where $\Delta|\eta|$ is positive or negative. Pseudorapidity is defined as $\eta=-\ln [\tan (\theta / 2)]$, where the polar angle $\theta$ is defined with respect to the LHC beamline. Some BSM models (such as those with new vector boson exchange in the $s$-channel) [11] predict different behavior in the lepton-based charge asymmetry $A_{C}^{\ell \ell}$ from the $t \bar{t}$ charge asymmetry, so it is interesting to measure both charge asymmetries.

In the combined $e e, e \mu$, and $\mu \mu$ channels, the lepton-based asymmetry is measured to be $A_{C}^{\ell \ell}=$ $0.023 \pm 0.012$ (stat.) \pm 0.008 (syst.), and the $t \bar{t}$-based asymmetry is measured to be $A_{C}^{t \bar{t}}=0.057 \pm$ 0.024 (stat.) \pm 0.015 (syst.). The SM predictions are $A_{C}^{\ell \ell}=0.004 \pm 0.001$ and $A_{C}^{t \bar{t}}=0.006 \pm 0.002$, respectively. The main systematic uncertainties in this analysis result from uncertainties on the jet energy scale and resolution, pile-up, and signal modelling. This measurement is statistically limited, but consistent with the Standard Model prediction. 


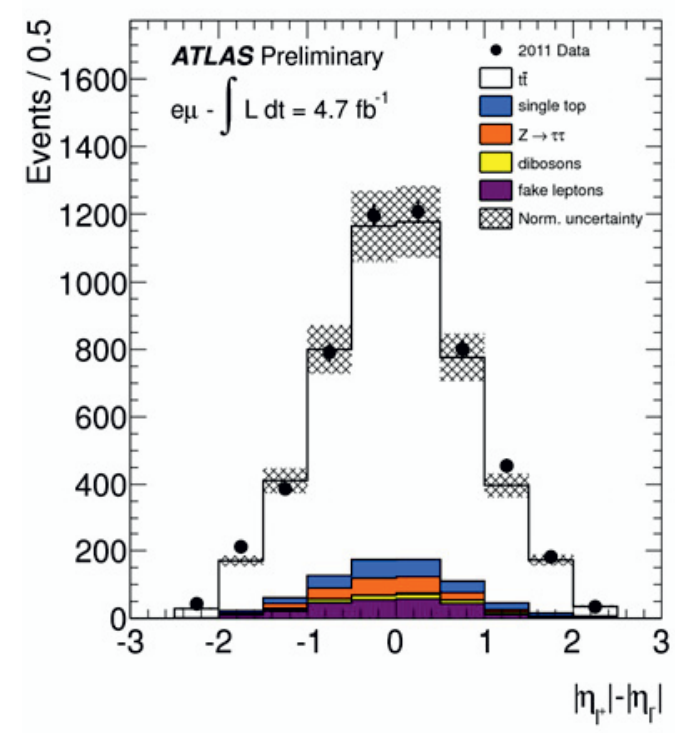

Figure 5. $\Delta|\eta|$ distribution for pairs of leptons in the $e \mu$ channel after selection. The black points are data, and the solid lines are Monte Carlo simulation. The shaded area marks the systematic uncertainties on the signal and background distributions [11].

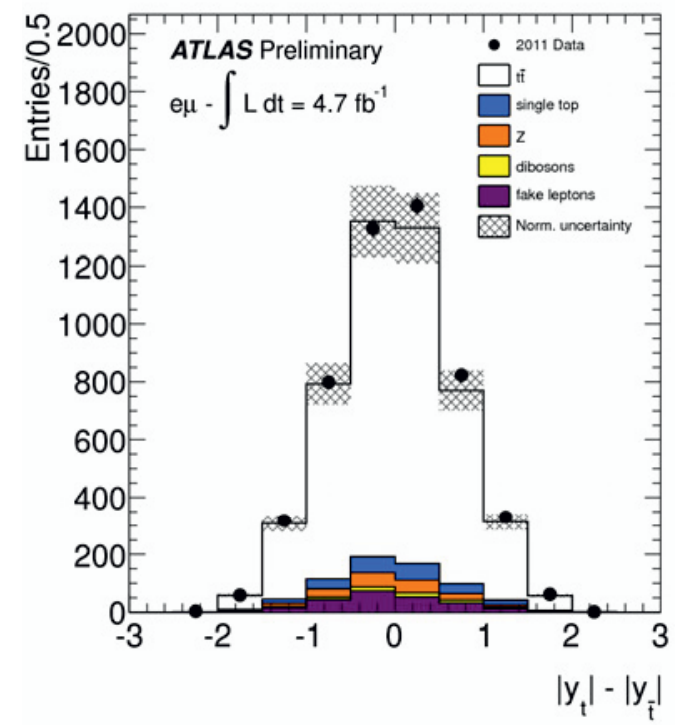

Figure 6. $\Delta|y|$ distribution for top quark pairs in the $e \mu$ channel. The black points are data, and the solid lines are Monte Carlo simulation. The shaded area marks the systematic uncertainties on the signal and background distributions [11].

\section{Search for $t \bar{t}+Z$ Boson Production}

A search for top pair production in association with a $Z$ boson was performed using $4.7 \mathrm{fb}^{-1}$ of $\sqrt{s}$ $=7 \mathrm{TeV}$ data collected in 2011 [12]. Candidate $t \bar{t}+Z$ events were selected with 3 leptons (electrons or muons), more than $30 \mathrm{GeV}$ of missing transverse energy, and at least 4 jets (at least one of which is $b$-tagged). At least one pair of leptons must have the same flavor and opposite charges, and have an invariant mass within $10 \mathrm{GeV}$ of the $Z$ boson mass. Figure 7 shows the invariant mass of the pair of opposite-sign, same-flavor leptons from the $Z$ boson decay. The other lepton comes from the top decay $t \rightarrow W b$, where the $W$ boson then decays leptonically.

A cut and count analysis was performed to measure the $t \bar{t}+Z$ cross section, $\sigma_{t \bar{t} Z}$, and the matrix method [13] was used to identify fake leptons. Fake lepton backgrounds from $Z+$ jets events and from events that do not contain a $Z$ boson were estimated separately.

One event was observed in the data (see Figure 8), while the number of expected signal events was $0.85 \pm 0.04$ (stat.) \pm 0.14 (syst.) and the number of expected background events was $0.28 \pm 0.05$ (stat.) \pm 0.14 (syst.). An upper limit on the $t \bar{t}+Z$ cross section of $\sigma_{t \bar{t} Z}=0.71 \mathrm{pb}$ was set, at $95 \%$ confidence level. The SM prediction for the $t \bar{t}+Z$ cross section is $\sigma_{t \bar{t} Z}=0.14 \mathrm{pb}$. The dominant systematic uncertainty in this analysis is the background normalization. This measurement is consistent with the Standard Model prediction. 


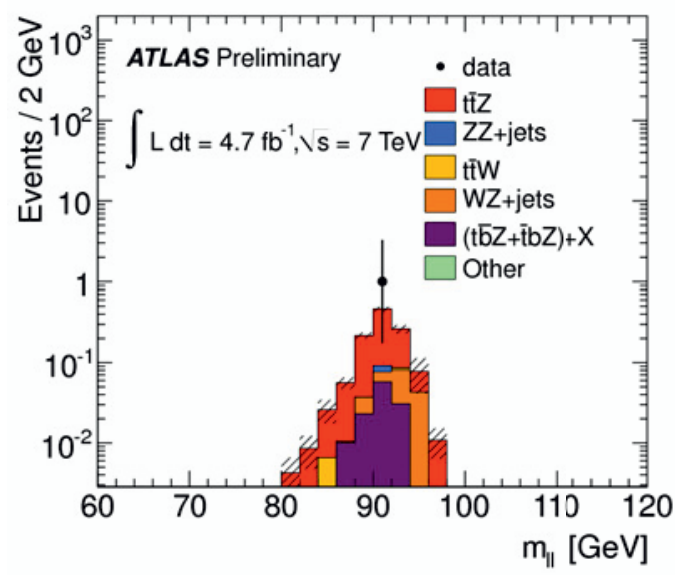

Figure 7. Invariant mass of the lepton pair originating from the $Z$ decay. The single observed candidate $t \bar{t}+Z$ event (black dot) is shown, with background Monte Carlo predictions (solid lines) [12].

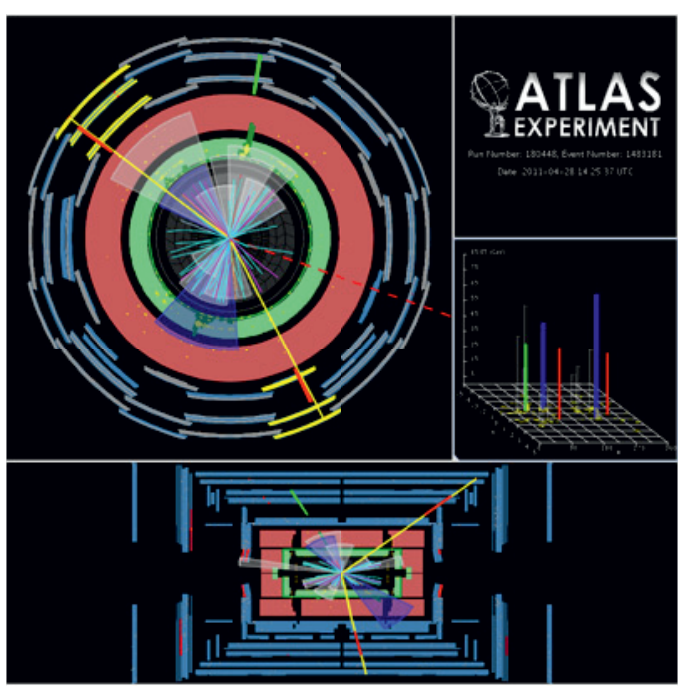

Figure 8. This event display shows the single observed $t \bar{t}+Z$ candidate event, with two oppositelycharged muons from the $\mathrm{Z}$ boson decay, one electron from the semileptonic $t \bar{t}$ decay, two jets, and missing transverse energy [12].

\section{Measurement of the $t \bar{t}+\gamma$ Production Cross Section}

A measurement of the cross section for events with a top pair and an additional photon in the final state was made using $1.04 \mathrm{fb}^{-1}$ of $\sqrt{s}=7 \mathrm{TeV}$ data collected in 2011 [14]. $t \bar{t}+\gamma$ candidate events were selected with one lepton (electron or muon), a large amount of missing transverse energy, at least 4 jets (at least one of which is $b$-tagged), and one good photon with transverse momentum $\left(p_{T}\right)>15$ GeV. Here, "good" implies that the photon candidate has fulfilled certain requirements on shower shape and hadronic leakage [15].

In this analysis, prompt photons (signal) are separated from photons that originate from hadron decays (hadron fakes) using a template fit method. A track-based isolation variable, $p_{T}^{\text {cone } 20}$, is used to

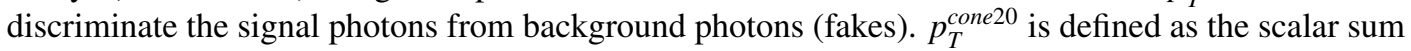
of the transverse momenta of all tracks in a cone with $\Delta R<0.2$, where $\Delta R=\sqrt{\Delta \eta^{2}+\Delta \phi^{2}}$.

As shown in Figure 9, prompt photons are generally very isolated ( $\operatorname{small} p_{T}^{\text {cone20 }}$ ), while the hadron fakes are surrounded by other particles from the fragmentation process (larger $p_{T}^{\text {cone20}}$ ). The templates used in this fit are derived from data.

A cut and count analysis was performed to measure the $t \bar{t}+\gamma$ cross section times branching ratio, where the branching ratio accounts for $t \bar{t}+\gamma$ events with photon $p_{T}>8 \mathrm{GeV}$, and single- or dilepton $t \bar{t}$ events. 52 (70) events were observed in the electron (muon) channel, which results in the cross section time branching ratio measurement $\sigma_{t \bar{t}+\gamma} \cdot B R=2.0 \pm 0.5$ (stat.) \pm 0.7 (syst.) \pm 0.08 (lumi.) pb. The Standard Model prediction for this cross section times branching ratio is $2.1 \pm 0.4 \mathrm{pb}$. The largest systematic uncertainties are from initial and final state radiation, the jet energy scale, and photon identification. This measurement excludes the background-only hypothesis at $2.7 \sigma$. 

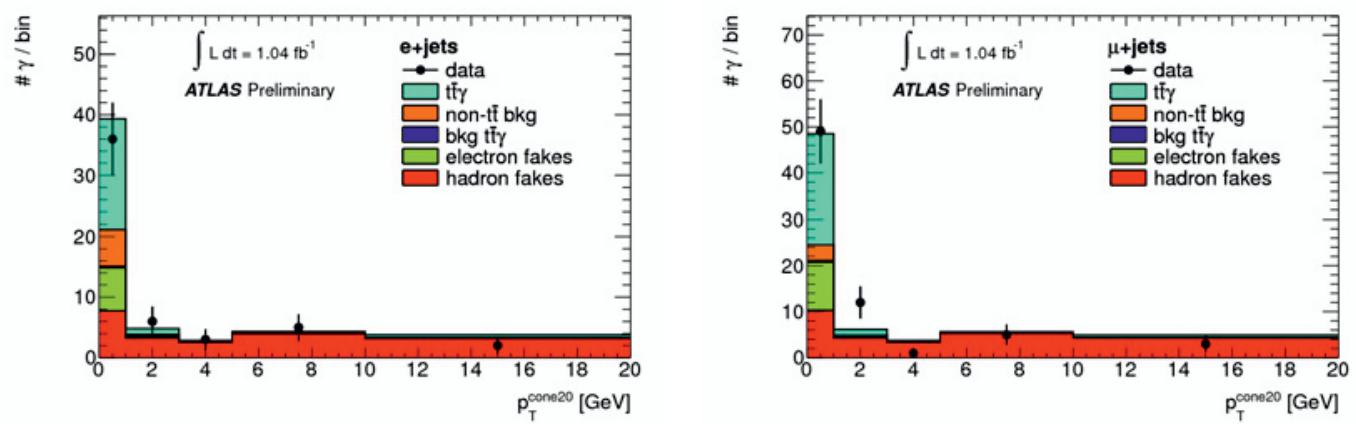

Figure 9. Track-based isolation of the photon candidates in the electron channel (left) and muon channel (right) [14].

\section{Conclusion}

The $\sqrt{s}=7 \mathrm{TeV}$ dataset collected in 2011 with the ATLAS detector at the Large Hadron Collider was used to measure many properties of top pair events. The following analyses were presented here:

- The measurement of spin correlation in top pair events is consistent with the Standard Model prediction, and excludes the no-correlation hypothesis at more than $5 \sigma$.

- Two measurements of charge asymmetry were presented. In the single-lepton channel, inclusive as well as differential charge asymmetry measurements were made, while in the dilepton channel, only an inclusive measurement was made. Both are consistent with the Standard Model predictions.

- An upper limit was set on the cross section for $t \bar{t}$ production in association with a $Z$ boson. That limit is consistent with the Standard Model cross section.

- Finally, a measurement was made of the cross section for $t \bar{t} \gamma$ production, which excluded the background-only hypothesis with a significance of $2.7 \sigma$.

These studies show no evidence for new physics in top pair events. There are updates coming for these analyses, which will make use of the more than $20 \mathrm{fb}^{-1}$ of data available in the $\sqrt{s}=8 \mathrm{TeV}$ 2012 dataset, as well as novel analytical approaches.

\section{References}

[1] Particle Data Group, Phys. Rev. D 86, 010001 (2012).

[2] ATLAS Collaboration, JINST 3, S08003 (2008).

[3] W. Bernreuther and Z. G. Si, Nucl. Phys. B 837, 90 (2010).

[4] ATLAS Collaboration, Phys. Rev. Lett. 108, 212001 (2012).

[5] P. Uwer, Phys. Lett. B 609, 271 (2005).

[6] S. Frixione and B. R. Webber, JHEP 06, 029 (2002).

[7] G. Corcella et al., JHEP 01, 010 (2001).

[8] W. Bernreuther and Z. G. Si, Phys. Rev. D 86, 034026 (2012).

[9] ATLAS Collaboration, ATLAS-CONF-2013-078, http://cds. cern. ch/record/1562978.

[10] G. Choudalakis, arXiv:1201.4612. 
EPJ Web of Conferences

[11] ATLAS Collaboration, ATLAS-CONF-2012-057, http://cds . cern. ch/record/1453785.

[12] ATLAS Collaboration, ATLAS-CONF-2012-126, http://cds.cern. ch/record/1474643.

[13] ATLAS Collaboration, Eur. Phys. J. C 711577 (2011).

[14] ATLAS Collaboration, ATLAS-CONF-2011-153, http://cds.cern.ch/record/1398197.

[15] ATLAS Collaboration, Phys. Rev. D 85012003 (2012). 